\title{
Application and Consideration of MOOC Use in Animal Medicine Teaching
}

\author{
Tuerxunjiang Kuerban ${ }^{1 a}$, Dayong Tao ${ }^{1 b}$, Bo Jing ${ }^{1 c}$, Jianzhong $\mathrm{He}^{1 \mathrm{~d}}$, Haihong \\ Jiao $^{1 \mathrm{e}}$, Donghai Zhou ${ }^{2 f}$, Dexin Qiu ${ }^{1,2 g}$ and Dingzong Guo ${ }^{1,2 \mathrm{~h}^{*}}$
}

${ }^{1}$ College of animal science, Tarim university, Alaer City, XinJiang Uygur Autonomous Region, PRC, $843300,{ }^{2}$ College of animal medicine, Huazhong Agricultural University, Wuhan, HuBei province, PRC, 430070

*hlgdz@163.com

Keywords: MOOC; Educational model; Unidirectional ecological attribute; Anti-MOOC;Animal medicine teaching

\begin{abstract}
Objective: In recent years, massive open online course (MOOC) has rapidly become a novel type of remote educational model worldwide. In this article, MOOC was applied in the course teaching of veterinary medicine to investigate the unidirectional ecological attribute of MOOC. Methods: Classification comparison method was adopted to analyze the characteristics, advantages and relationship between MOOC and traditional classroom course, MOOC and national quality course, MOOC and flipped classroom course, virtual stimulation technology and micro-course. Results: MOOC was an excellent supplement of traditional classroom course, which contributed to fostering the innovative thinking and self-study capability of students, and promoting the interaction and exchange between teachers and students. In MOOC, computer technology was utilized to collect the complex and difficult cases, zoonotic cases and rare cases and design these cases into corresponding modules. The students could access to the corresponding case by clicking the module to fill out the vacancy and eliminate the risk of diseases. MOOC resource website was not only available for resource release and download, but also for promoting the interaction between teachers and students in a specific module, which offered convenience for remote experimental practice for students. MOOC was a pivotal part of educational system after graduation from university. The findings in this study prompted that the relationship between MOOC and alternative types of educational models should be properly resolved, striving to achieve interconnection, intercommunication and cooperation. Conclusions: MOOC is an excellent supplement of traditional classroom course, which cultivates the innovative thinking, self-learning ability and remote study of students, and promotes the teacher-to-student interactions.
\end{abstract}

\section{Introduction}

Since its emergence, massive open online course (MOOC) has encountered two sharply divergent arguments. The proponents consider that MOOC predicts the possibility of revolutionary changes in the educational field, and even substitutes the traditional university and disrupts the conventional pattern of higher education. Instead, the opponents propose that MOOC fails to achieve substantial breakthrough and merely acts as another version of television education. At present, the teaching concept of online courses including MOOC has not been completely realized. The drawbacks of traditional teaching remain unresolved and no concrete changes have been obtained. In addition, MOOC does not escape from the teacher-centered teaching pattern in which the students passively learn what is taught by the teachers ${ }^{[1]}$. The role and effect of MOOC in classroom course remain to be investigated. In this study, MOOC was applied in the teaching course of veterinary medicine, aiming to explore the utilization and progression of MOOC in teaching courses. 


\section{Application of MOOC in Teaching Course of Veterinary Medicine}

Application of MOOC in Classroom Course of Veterinary Medicine. Veterinary medicine is a demanding course theoretically and in practice ${ }^{[2]}$. Although MOOC fails to substitute classroom teaching course, it still plays an influential role in the following aspects. (1) As a supplement or material of classroom course, MOOC can diversify the course content. For instance, the relationship between the morphology and function of a tissue organ can be realized by a teaching module, which is vivid, appropriate and saves teaching time. (2) MOOC is applicable to the individualized educational model, which contributes to the screening and cultivation of the innovative talents. During MOOC course, the students can simply choose the content and progression of the study courses according to individual learning status, which discards the conventional binding and level educational models and promotes the development of the innovative talents. (3) MOOC is a convenient approach to evaluate the learning and teaching effects and promote the interaction between teachers and students. In the module design of MOOC, a test module is designed in which the homework can be arranged in each module or after multi-module learning. It not only facilitates self-testing for the students, but also assists the teachers to understand the understanding level of the students for the course content. Examination module: mid-term and final examinations can be arranged according to corresponding requirement. In the teacher-to-student interaction module, teachers can communicate with students to resolve the common or individual questions on MOOC platform. Students can raise a question regarding the course content, exchange feelings and experience, and communicate with classmates. (4) MOOC is applicable to the teaching of course content related to complex and abstract thinking. Due to the time and source material limits, it is challenging to teach relevant course contents in a fixed period of time, such as the network regulation of a certain disease, internal relationship between pathogenesis and clinical symptoms, and information transmission and excitation-contraction coupling at the myconeutral junction. Nevertheless, teachers are capable of completing the teaching task of such course contents by using the virtual stimulation technology.

Application of MOOC in Teaching of Experimental and Social Practice in Veterinary Medicine. Veterinary medicine is a practical and empirical medicine field. The quantity and content of the experiment and practice directly affect the quality of the student cultivation. MOOC can accumulate the experiment, practice and clinical experience through multiple pathways. In MOOC, computer technology is utilized to collect the complex and difficult cases, zoonotic cases and rare cases and design these cases into corresponding modules. The students can access to the corresponding case by clicking the module to fill out the vacancy and eliminate the risk of diseases. During social practice and teaching in a site distant from schools, it is difficult to perform clinical examination and diagnosis. Therefore, students can simply utilize the MOOC to learn relevant knowledge. Moreover, students can also upload the video of specific cases, raise questions and seek assistance and guidance from teachers to resolve their confusing questions. As a free and open-access online curriculum, MOOC can supply diverse course contents, such as case video, extra-curricular reading materials and knowledge examination module. Besides, MOOC also establish an online interaction platform for the professors and students. MOOC resource website is not only available for resource release and download, but also for promoting the interaction between teachers and students in a specific module, which offers convenience for remote experimental practice for students ${ }^{[3,4]}$.

Application of MOOC in the Education after Graduation from University. Continuing education after graduation from university is a pivotal component of the entire education system in China ${ }^{[5]}$. More and more social practitioners with higher education certificate realize the importance of knowledge update and curriculum training after graduation to adapt to the swift development of science and technology in modern society, especially the new instruments, equipments, technologies, medicines and theories in the field of veterinary medicine ${ }^{[6]}$. The bachelors are fully aware of their knowledge system, have a strong desire for learning and establish a explicit target. However, they have no time to attend classroom course and receive face-to-face guidance from teachers. MOOC offers sufficient time and space for the bachelors and sets up a 
platform for continuing education. Taken together, MOOC is free and open-access platform for anyone regardless of region, age and certificate. Once registered and login the MOOC, any student can select and learn the courses they are interested in. Different from the quality courses, MOOC consists of various modules and is totally free for all students. Students can simply select any module according to individual interests and requirements. Flexible performance assessment is another appealing point for the adults. A portion of students merely focus on personal growth rather than academic score and course certificate. For those who require performance assessment, curriculum, project and credit certificates can be established ${ }^{[7]}$.

\section{Thinking of MOOC construction}

Integrated Development of MOOC and National Quality Course. During the period of 11th and 12th five-year plans, tremendous time and energy have been injected to establish over 30000 school, provincial and national quality courses $(n=5727){ }^{[8]}$. However, the teaching effect of these quality courses falls short of expectation. Partial quality courses are limited to the titles and awards. The course content is obsolete and slowly updated. Due to the insufficient learning platform, teachers and students can not smoothly communicate and exchange. The link of $35 \%$ of quality course homepages is down. The utilization rate of teaching courses is low ${ }^{[9]}$. Both MOOC and quality courses are remote and open-access courses. Thus, MOOC can be utilized to increase the utilization rate of quality courses. The advantages of MOOC can resolve the disadvantages of quality courses. Application of MOOC pattern to re-utilize the quality courses is not only viable theoretically, but also feasible in reality. Previous investigations have demonstrated that MOOC and quality courses have common targets and specific emphases. The construction of national quality course proposes the objective of the high-quality teaching staff, course content, teaching method, teaching material and teaching management ${ }^{[10]}$. Although the objective of MOOC has not been proposed, MOOC surpasses the national quality courses in terms of the construction contents and technical standards. National quality courses aim to attain the parameters above through curriculum construction. Instead, MOOC emphasizes the concrete contents of curriculum construction, the superiority, utilization, interaction and open-access ${ }^{[11]}$. National quality courses and MOOC significantly differ in the curriculum standards and requirements. Quality courses emphasize the system and integrity of curriculum content. The teaching content of each chapter and the teaching video of each class should be relatively integrated. Each MOOC curriculum consists of multiple modules for 10 to 20 hours. Each module requires curriculum content for 1 to 2 hours, which is separated into different classes, 5-15 min video for each class. Each video materials analyze one knowledge point (concept, mechanism and topic, etc.). One or two class tests are designed for each video demonstration. Students can also answer these questions in the test module after class ${ }^{[12]}$. It is technologically feasible to establish a sharing platform between MOOC and quality courses, whereas it should be approved by the upper authority.

Integration of MOOC other Advanced Technologies (Virtual Stimulation Technology and Flipped Classroom Course). Computer technology significantly reduces the cost of information transmission. The modernization of digital compression technology allows for higher compatibility of the internet-based transmission system. Better loading capability provides a technical support for the integration among multiple courses. In contemporary remote teaching mode, besides MOOC and quality courses, virtual stimulation technology, flipped classroom and micro-course have emerged in recent years. These technologies and courses collectively aim to serve remote teaching, whereas have different targets ${ }^{[13]}$. Virtual stimulation technology is an excellent supplement of MOOC to obtain three dimensional images. Visual, auditory and sensory channels are employed to deliver virtual interaction, which significantly elevates the video quality, appreciation and appealing of MOOC. It is more vivid and interesting to apply virtual simulation experiment, MOOC, flipped classroom and micro-course to the animal experiment and clinical diagnosis of veterinary medicine. To attain the goal of integrated development and reasonable utilization of resources, the thinking mode should be changed. The concept of integrated development should be resolved from the upper authority (national Ministry of Education) to implement platform share, diverse development and 
public-oriented service. Secondly, technical, management and operation integration should be delivered to realize the goal of interconnection and intercommunication.

Unidirectional Ecological Attribute of MOOC. Ecology is a scientific analysis and study of interactions that organisms have with each other, other organisms, and with their environment. Classroom ecology utilizes ecological concept to study the interactions among middle school students, teachers and their environment. Ecology is essentially a discipline of interactions, which is consistent with MOOC. Although as a remote course, MOOC consists of teachers, students, course content, teaching method and means, physical environment and alternative ecological factors. MOOC still possesses the classroom ecological attribute ${ }^{[14]}$. The course content of MOOC is manufactured and displayed in advanced, whereas the students, course content, teaching method and means, and physical environment are actual and real, suggesting that the verbal and behavioral manner of teachers, course content and teaching capability can affect the learning effect of students. Nevertheless, learning attitude and effect of students do not exert effects upon teachers as the classroom courses. The teacher-to-student interaction can be realized merely through the MOOC platform. This asymmetric feature of communication is known as the unidirectional ecological attribute of MOOC in this investigation. It severely impedes the development and public benefits of MOOC, which remains to be urgently resolved in subsequent research.

Anti-MOOC Issues During the Construction of MOOC. In 2013, anti-MOOC course was proposed by Audrey Waters to target the problems of MOOC. MOOC is a global online open-access curriculum in which thousands of students from different regions can learn the MOOC courses and deliver discussions simultaneously. However, it is very difficult for MOOC to achieve teacher-to-teacher, student-to teacher and student-to-student interactions. The objective of anti-MOOC is not to oppose MOOC but to resolve the problems of MOOC. To counteract this issue, Audrey Waters proposed the concept of small online course to restrict the course scale. Specifically, the course scale is accessible to dozens to hundreds of students. Only candidates eligible for certain standards can be allowed to learn the MOOC courses. At present,Some restricted online course are being tentatively explored in Harvard University. From this perspective, a majority of MOOC in China belongs to small online courses, and merely a small portion of MOOC is accessible to a large quantity of students, which is an inevitable issue to be resolved towards the goal of real MOOC.

\section{Acknowledgements}

Supported by a project grant from National Teaching Demonstration Center construction project (Higher education letter of Ministry of Education No.[2013]10).

\section{References}

[1] S. W. Zhang, R. K. Liu. Debate and commentary about MOOC. Modern Distance Education, 2015(162)3-9.

[2] D. J. Dong, X. Y. Li, C.X. Yang, G. J. Qu, G. X. Hu, Q. S. Zhang. Construction of curriculum system for practice teaching fpr outstanding talents in animal science specialty-based on the case of Jilin Agricultural University. Heilongjiang Animal Science and Veterinary Medicine, 2016(1)157-159

[3] H. Zhang, X. M. Ren. Traditional combined with modern animal science for culture of talented veterinarians in urban. Higher Agricultural Education. 2016(4)60-61

[4] J. W. Chu, Y. Y. Li. Comparison of information resource and organization of international MOOC websites. Research on Library Science.2016(12)45-49

[5] X. X. Yang, H. C. Shen. Analysis of supply side and demand side of higher continuing education. Continuing Education.2017,254(1) 3-6

[6] B. G. Ou, Y. Z. Luo, H. J. Wu, Z. H. Li.Current situation and solutions to the problems in the graduate education of professional degree of veterinary medicine in China.Academic Degrees \& Graduate Education.2010(5)33-38. 
[7] Y. F. Tong, X. B. Cheng. Implementation strategy of MOOC in adult online study. Adult Education.2016,353(6)66-69

[8] W. W. Qin. Investigation of current status of 10-year development of national quality course.Distance Education in China,2013(8)53-57

[9] S. F. Wang. The main problems with the construction of quality course websites and their solutions.Journal of Higher Education Management.2011,5(4)57-6

[10]C. L. Li, S. T. Xu, H. L. Ren.The practice and exploration of quality course construction in agricultural universities - based on the case of the course "Plant Tissue and Cell Culture".Jiaoyu jiaoxue luntan.2017(8)117-9

[11]Y. G. Wang, Q. Zhang.MOOC:characteristics and study mechanism.Education Research.2014,416(9):112-11

[12]X. S. Tong, X. J. Jia. Construction and exploration of MOOC quality rating system.Distance Education in China.2015(5)63-71.

[13]H. Z. Hu, F. Guan, Q. Guo. Research and practice of teaching mode in colleges based on flipped classroom and MOOC.Experimental Technology and Management.2016,33(12) 189-192.

[14]C. L. Shen, X. Yuan. Unbalance and restructure of English course ecology in the context of information linguistic environment. Teaching and Management. 2016(2)102-1

[15] Y. Li, W. Chen, J. C. Liu. Exploration and practice on the teaching reform of literature retrieval course for postgraduate under the influence of the anti-mooc and the flipped classroom. Journal of Academic Libraries.2015(4)97-10. 